\title{
Worst-case comparison of valid inequalities for the TSP
}

\author{
Michel X. Goemans ${ }^{1}$ \\ Department of Mathematics, Room 2-382, Massachusetts Institute of Technology, Cambridge, MA \\ 02139-4307, USA
}

Received 17 June 1993; revised manuscript received 13 December 1994

\begin{abstract}
We consider most of the known classes of valid inequalities for the graphical travelling salesman polyhedron and compute the worst-case improvement resulting from their addition to the subtour polyhedron. For example, we show that the comb inequalities cannot improve the subtour bound by a factor greater than $\frac{10}{9}$. The corresponding factor for the class of clique tree inequalities is $\frac{8}{7}$, while it is $\frac{4}{3}$ for the path configuration inequalities.
\end{abstract}

Keywords: Polyhedral combinatorics; Valid inequalities; Travelling salesman; Worst-case analysis

\section{Introduction}

In the last decade, strong cutting-plane methods have successfully solved larger and larger symmetric travelling salesman problem (TSP) instances to optimality. In the early nineties, Padberg and Rinaldi [28] have solved optimally an instance with 2,392 cities, and more recently Applegate et al. [1] have solved optimally instances with 3,038, 4,461 and even 7,397 cities. These cutting-plane methods are based on an extensive investigation of the facets of the symmetric travelling salesman polytope (STSP) and its relatives. Many classes of facet-defining valid inequalities are now known for STSP, see $[3,5,7,9,12,15,17,22-24,26]$.

The success of cutting-plane methods for the TSP is however not fully understood. In particular, it is not clear which classes of inequalities are most or least useful in solving TSP instances. For obvious reasons of efficiency, the choice of the inequalities to use

\footnotetext{
${ }^{1}$ E-mail: goemans@math.mit.edu. Research supported in part by Air Force contract F49620-92-J-0125, DARPA contract N00014-92-J-1799 and NSF contract 9302476-CCR.
} 
in a cutting-plane algorithm is typically dictated by whether efficient exact or heuristic algorithms are known for the corresponding separation problem. For this reason, cuttingplane approaches tend to use, in addition to the subtour elimination constraints, the comb inequalities or the clique tree inequalities, see $[1,8,13,14,19,26-28]$. An exception is a recent implementation of [6] which is based on the path inequalities. The motivation of this paper is best expressed by the following quote from [24]:

For which ... inequalities ... would it be worth investing research time to find good separation algorithms, and computation time to run them in a cuttingplane algorithm? An answer to this question would be easier if there was some indicator that could be used to compare two classes of inequalities, with respect to their potential effectiveness in a polyhedral cutting-plane algorithm.

In this paper, we describe such an indicator by evaluating the worst-case improvement resulting from the addition of many classes of valid inequalities to the subtour polyhedron.

For the reader familiar with polyhedral studies of the TSP, Table 1 gives a preview of the results. The column "Strength" gives for various classes of inequalities the worst-case ratio between the values obtained by optimizing over the subtour polyhedron plus an additional class of inequalities and over the subtour polyhedron itself. The inequalities are assumed to be in graphical form and the worst case is taken over all nonnegative cost functions. In some sense, "worst-case" has to be understood as best-case, since the larger the improvement the better it is. The most notable entries in the table are the fact that clique tree inequalities cannot improve the subtour polytope bound by more than a factor of $\frac{8}{7}$, while the path configuration inequalities improve it in the worst case by $\frac{4}{3}$.

The paper is structured as follows. Section 2 reviews some well-known polyhedral results for the TSP. In Section 3, we formally describe a measure to compare two polytopes and show how it can be used to compare various relaxations of the TSP. The results summarized in Table 1 are established in Section 4 and interpreted in Section 5. Finally, we conclude with some remarks in Section 6.

\section{Background material}

We assume a basic knowledge of polyhedral combinatorics, especially related to the travelling salesman problem. The reader is referred to $[16,29]$ for a very thorough introduction.

The symmetric travelling salesman polytope (STSP) is defined as the convex hull of incidence vectors of Hamiltonian cycles. Optimizing any cost function over STSP is equivalent to finding a Hamiltonian cycle of minimum cost. There are several drawbacks associated with the polytope STSP. First, it is not full-dimensional and this makes its study more difficult. Also, in our worst-case analysis, it will be crucial to consider any nonnegative cost function, while one typically considers instances of the TSP for 
Table 1

Strength of TSP inequalities

\begin{tabular}{|c|c|c|c|}
\hline Class of inequalities & & Strength & Reference \\
\hline \multirow[t]{2}{*}{ Comb } & with $t$ teeth & $\frac{3 t+1}{3 t}$ & Corollary 5 \\
\hline & in general & $\frac{10}{9}$ & Corollary 5 \\
\hline \multirow[t]{3}{*}{ Clique tree } & with $h$ handles and $t$ teeth & $\frac{3 t+2 h-1}{3 t+h-1}$ & Theorem 4 \\
\hline & with $h$ handles & $\frac{8 h+2}{7 h+2}$ & Corollary 5 \\
\hline & in general & $\frac{8}{7}$ & Corollary 5 \\
\hline \multirow[t]{2}{*}{ Path configuration } & with $k$ paths & $\frac{k+1}{k}$ & Theorem 6 \\
\hline & in general & $\frac{4}{3}$ & Theorem 6 \\
\hline \multirow[t]{2}{*}{ Crown } & with parameter $k$ & $\frac{6 k(k-1)-1}{6 k(k-1)-k}$ & Theorem 7 \\
\hline & in general & $\frac{11}{10}$ & Theorem 7 \\
\hline \multirow[t]{2}{*}{ Hypohamiltonian } & on $k$ vertices & $\leqslant \frac{k+1}{k}$ & Theorem 8 \\
\hline & in general & $\frac{11}{10}$ & Theorem 8 \\
\hline
\end{tabular}

which the cost function satisfies the triangle inequality. To avoid both drawbacks, we will allow multiple visits of a vertex and consider the problem of finding an Eulerian subgraph (or multigraph) of minimum cost and its associated polyhedron. Given a graph $G=(V, E)$, an Eulerian sub(multi) graph ${ }^{2}$ is a collection of edges of $E$ (with possible repetition) such that the degree of every vertex is even and the subgraph is connected. For any cost function, the cost of the minimum Eulerian subgraph is equal to the value of the optimum Hamiltonian cycle with respect to the shortest path costs. The convex hull of the incidence vectors of Eulerian subgraphs is called the graphical travelling salesman polyhedron (GTSP). This polyhedron was introduced in [7,9] and was further investigated in [10,22-25]. GTSP is full-dimensional since it is of blocking type, i.e., if $x \in \mathrm{GTSP}$ and $y \geqslant x$, then $y \in$ GTSP.

Many classes of facet-defining valid inequalities are known for GTSP. The simplest is the class of subtour elimination constraints (also called loop constraints in [16] or cocycle inequalities in [25]): $x(\delta(S)) \geqslant 2$ for any $S \subset V$, where $x(F)=\sum_{e \in F} x_{e}$ and $\delta(S)$ represents the coboundary of $S$ defined as $\{e=(i, j):|S \cap\{i, j\}|=1\}$. Naddef and Rinaldi [25] have shown that any facet-defining inequality for STSP gives rise to a facet-defining inequality for GTSP. For this purpose, one needs to convert the valid inequality for STSP into tight triangular form by adding or subtracting multiple of the degree constraints $x(\delta(\{i\}))=2$. An inequality $a x \geqslant a_{0}$ is in tight triangular form [25] if

\footnotetext{
${ }^{2}$ They are also called tours or closed walks, see $[7,25]$.
} 
(1) the nonnegative coefficients $a_{e}$ satisfy the triangle inequality $a_{i j}+a_{j k} \geqslant a_{i k}$ for all $i, j, k$, and

(2) for all $j \in V$, there exists $i, k \in V-\{j\}: a_{i j}+a_{j k}=a_{i k}$.

Naddef and Rinaldi [25] prove that any nontrivial ${ }^{3}$ tight triangular facet-defining inequality for STSP is also a facet-defining valid inequality for GTSP. Furthermore, it is easy to see that every facet-defining inequality for GTSP, except the nonnegativity constraints and the subtour elimination constraints corresponding to singleton sets, must be in tight triangular form [25]. The most well-known class of inequalities for STSP (and thus also for GTSP when in tight triangular form) is the clique tree inequalities introduced in [17]. They generalize the comb inequalities of [5] and their extensions [15]. Many other classes of inequalities are known for STSP or GTSP, including the path inequalities [7], the path tree inequalities [23], the binested inequalities [22], the crown inequalities [24] and the rank inequalities [12].

The simplest relaxation of GTSP consists of the subtour elimination constraints. The corresponding subtour polyhedron SP, or subtour elimination polyhedron, is defined as

$$
\begin{aligned}
& \mathrm{SP}=\left\{x \in \mathbb{R}^{|E|}: x(\delta(S)) \geqslant 2, S \subset V,\right. \\
& \left.x_{e} \geqslant 0, \quad e \in E\right\} \text {. }
\end{aligned}
$$

From an observation of Cunningham [21] (see [11]), it follows that optimizing a nonnegative cost function over SP is equivalent to optimizing the shortest path cost function over the subtour polytope, obtained by adding the degree constraints $x(\delta\{i\})=$ 2 for all $i \in V$ to SP. As a result, the value obtained by optimizing over SP is exactly equal to the Held-Karp lower bound [18]. Interpreting their result in terms of GTSP, Wolsey [31] and Shmoys and Williamson [30] have shown that

$$
\frac{\operatorname{Min}\{c x: x \in \mathrm{GTSP}\}}{\operatorname{Min}\{c x: x \in \mathrm{SP}\}} \leqslant \frac{3}{2}
$$

for any nonnegative cost function $c$. However, this bound does not appear to be tight and, in fact, the following conjecture motivated this study (see Sections 3 and 5).

Conjecture 1. For any nonnegative cost function $c$,

$$
\frac{\operatorname{Min}\{c x: x \in \mathrm{GTSP}\}}{\operatorname{Min}\{c x: x \in \mathrm{SP}\}} \leqslant \frac{4}{3} \text {. }
$$

Instances are known which achieve a bound of $\frac{4}{3}$. One can obtain stronger relaxations of GTSP by adding to SP some of the classes of inequalities mentioned previously.

\section{Worst-case comparison of relaxations}

If $P$ and $Q$ are polyhedra in $\mathbb{R}^{n}$, then we say that $P$ is a relaxation of $Q$ or $Q$ is a strengthening of $P$ if $P \supseteq Q$. Given a relaxation $P$ of a polyhedron $Q$, we are

\footnotetext{
${ }^{3}$ Not defining the same inequality as $x_{e} \geqslant 0$ for any edge $e$.
} 
interested in evaluating how well does $P$ approximate $Q$. In the context of polyhedral combinatorics, this question arises in two different ways. Under the most classical perspective, $Q$ is a combinatorial optimization polyhedron and one would like to estimate how tight is a given relaxation $P$ of $Q$. Another possible scenario is that one has a standard relaxation $P$ of some combinatorial optimization polyhedron $R$ and one would like to evaluate the improvement that would result from the addition to $P$ of a certain class of valid inequalities. So, in the latter case, one has two relaxations $P$ and $Q$ of $R$ where $Q$ is a strengthening of $P$.

How does one compare two relaxations? This question is not well understood and, in fact, one can formulate several notions of "tightness". For a recent investigation on a notion based on the volumes of $P$ and $Q$, see [20]. In this paper, we adopt an optimization point of view.

Throughout this section, we assume that $P \subseteq \mathbb{R}_{+}^{n}$ is a relaxation of a polyhedron $Q \subseteq \mathbb{R}_{+}^{n}$ of blocking type. This assumption leads to a very natural notion of tightness. Given $\alpha \geqslant 1$, we say that $P$ is an $\alpha$-relaxation of $Q$ or $Q$ is an $\alpha$-strengthening of $P$ if $Q \supseteq \alpha P=\{\alpha x: x \in P\}$, i.e., $Q$ is a relaxation of $\alpha P$. Any $\alpha$-relaxation is also a $\beta$-relaxation for any $\beta \geqslant \alpha$. Also, let $t(P, Q)$ denote the minimum value of $\alpha$ such that $P$ is an $\alpha$-relaxation of $Q$. Notice that $t(P, Q) \geqslant 1, t(P, Q)=1$ iff $P=Q$, and that $t(P, Q)$ could be infinite.

The parameter $t(P, Q)$ can be interpreted from an optimization point of view. Let $c$ be any vector in $\mathbb{R}_{+}^{n}$. If $P$ is an $\alpha$-relaxation of $Q$, then clearly

$$
\operatorname{Min}\{c x: x \in Q\} \leqslant \alpha \operatorname{Min}\{c x: x \in P\} .
$$

From the separating hyperplane theorem, the polyhedron $Q$ contains the polyhedron $\alpha P$ iff, for any cost vector $c$,

$$
\operatorname{Min}\{c x: x \in Q\} \leqslant \alpha \operatorname{Min}\{c x: x \in P\} .
$$

For a vector $c$ which fails to be nonnegative, this inequality is vacuous and, therefore, the converse statement also holds, as stated in the following lemma.

Lemma 2. Let $P$ be a relaxation of a polyhedron $Q$ in $\mathbb{R}_{+}^{n}$ of blocking type. Then $P$ is an $\alpha$-relaxation of $Q$ iff, for any nonnegative vector $c \in \mathbb{R}^{n}$,

$$
\operatorname{Min}\{c x: x \in Q\} \leqslant \alpha \operatorname{Min}\{c x: x \in P\} .
$$

As a corollary, $t(P, Q)$ is equal to

$$
t(P, Q)=\operatorname{Sup}_{c \in \mathbb{R}_{+}^{n}} \frac{\operatorname{Min}\{c x: x \in Q\}}{\operatorname{Min}\{c x: x \in P\}},
$$

where, by convention, $\frac{0}{0}=1$.

The following result gives an alternate characterization of $t(P, Q)$ when a description of $Q$ in terms of linear inequalities is known. 
Theorem 3. Let $P$ be a relaxation of a polyhedron $Q$ in $\mathbb{R}_{+}^{n}$ of blocking type where $Q=\left\{x:^{*} a_{i} x \geqslant b_{i}\right.$ for $\left.i=1, \ldots, m\right\}, a_{i} \geqslant 0, b_{i} \geqslant 0$ for $i=1, \ldots, m$. Then,

$$
t(P, Q)=\operatorname{Max}_{i} \frac{b_{i}}{d_{i}},
$$

where $d_{i}=\operatorname{Min}\left\{a_{i} x: x \in P\right\}$.

Proof. From (1), it is clear that

$$
t(P, Q) \geqslant \operatorname{Max}_{i} \frac{\operatorname{Min}\left\{a_{i} x: x \in Q\right\}}{\operatorname{Min}\left\{a_{i} x: x \in P\right\}} \geqslant \operatorname{Max}_{i} \frac{b_{i}}{d_{i}} .
$$

We therefore need to prove the reverse inequality.

Let $c$ be any nonnegative cost function. By strong duality, we know that

$$
\begin{aligned}
& \operatorname{Min} c x=\operatorname{Max} b^{\mathrm{T}} y \\
& \text { s.t. } A x \geqslant b \text { s.t. } A^{\mathrm{T}} y=c^{\mathrm{T}} \text {, } \\
& y \geqslant 0 \text {, }
\end{aligned}
$$

where "T" denotes the transpose. Let $y^{*}$ be the optimal dual solution of the above program. Then,

$$
\begin{aligned}
& \text { Min } c x=\operatorname{Min}\left(y^{*}\right)^{\mathrm{T}} A x \geqslant \sum_{i}\left\{\begin{array}{c}
\operatorname{Min} a_{i} x \\
\text { s.t. } x \in P
\end{array}\right\} y_{i}^{*}=\sum_{i} d_{i} y_{i}^{*} . \\
& \text { s.t. } x \in P \quad \text { s.t } \quad x \in P
\end{aligned}
$$

Hence,

$$
\frac{\operatorname{Min}\{c x: x \in Q\}}{\operatorname{Min}\{c x: x \in P\}} \leqslant \frac{\sum_{i} b_{i} y_{i}^{*}}{\sum_{i} d_{i} y_{i}^{*}}=\sum_{i}\left(\frac{d_{i} y_{i}^{*}}{\sum_{j} d_{j} y_{j}^{*}}\right) \frac{b_{i}}{d_{i}} .
$$

Since $d_{i} \geqslant 0$ (because $a_{i} \geqslant 0$ and $P \subseteq \mathbb{R}_{n}^{+}$) and $y_{i}^{*} \geqslant 0$, the latter quantity can be interpreted as a convex combination of $b_{i} / d_{i}$ and is therefore less or equal to

$$
\operatorname{Max}_{i} \frac{b_{i}}{d_{i}} \text {. }
$$

The result is proved by taking the supremum over all nonnegative cost functions $c$.

We should point out that we have not used the fact that $P$ is a relaxation of $Q$ in the above theorem.

Theorem 3 can be rephrased as follows. To compute $t(P, Q)$, one only needs to consider the cases in which a single inequality of $Q$ is added to $P$. This motivates the following definition. The strength of an inequality $a x \geqslant b$ with respect to a polyhedron $P$ is defined as

$$
\frac{b}{\operatorname{Min}\{a x: x \in P\}} \text {. }
$$

Theorem 3 implies that $t(P, Q)$ is equal to the maximum strength with respect to $P$ of a facet-defining inequality for $Q$. 


\subsection{Comparison of TSP relaxations}

In this paper, we evaluate $t$ (SP, $Q$ ) for many relaxations $Q$ of GTSP, i.e., we evaluate the worst-case improvement due to the use of the relaxation $Q$ instead of the subtour polyhedron SP. From Theorem 3, we know that $t(\mathrm{SP}, Q)$ is equal to the maximum strength (with respect to SP) of any facet-defining inequality for $Q$. In particular, Conjecture 1 , which says that $t$ (SP, GTSP) is at most $\frac{4}{3}$, is equivalent to the conjecture that the strength of any facet-defining valid inequality for GTSP is at most $\frac{4}{3}$.

Given a valid inequality $a x \geqslant b$ for GTSP, we need to compute $\operatorname{Min}\{a x: x \in \mathrm{SP}\}$ in order to evaluate its strength. By strong duality, $\operatorname{Min}\{a x: x \in \mathrm{SP}\}$ is equal to

$$
\begin{array}{ll}
\operatorname{Max} 2 \sum_{S} y_{S} \quad \text { subject to } \\
\sum_{S: e \in \delta(S)} y_{S} \leqslant a_{e}, \quad e \in E, \\
y_{S} \geqslant 0, & S \subset V .
\end{array}
$$

Notice that if $y$ is a feasible solution to (D), then $b /\left(2 \sum_{S} y_{S}\right)$ is an upper bound on the strength of the inequality $a x \geqslant b$. In the next section, we derive upper bounds on the strength of many valid inequalities for GTSP by appropriately constructing simple dual feasible solutions. The resulting upper bounds can in most cases be attained.

We should point out that two other notions of "strength" of TSP facet-defining inequalities have been proposed in [24]. As a first measure, Naddef and Rinaldi suggest to evaluate the number of Hamiltonian cycles on the corresponding facet. Their second measure is somewhat similar to our notion of strength. For an inequality $a x \geqslant b$ they evaluate the distance between the optimum solution to $\operatorname{Min}\{a x: x \in \mathrm{SP}, x(\delta(\{i\}))=2$ for all $i\}$ and the affine space defined by $\{x: a x=b, x(\delta(\{i\}))=2$ for all $i\}$. The degree constraints are present in the definition of the affine space to avoid that the measure depends on the representation of the facet in terms of a linear inequality.

\section{Strength of TSP inequalities}

The results of this section are summarized in Table 1.

\subsection{Clique tree inequalities}

Clique tree inequalities were discovered by Grötschel and Pulleyblank [17]. A clique tree $C$ consists of a collection of subsets of vertices partitioned into handles $H_{1}, \ldots, H_{h}$ and teeth $T_{1}, \ldots, T_{t}$ such that

(1) no two teeth intersect,

(2) no two handles intersect,

(3) each tooth has at least one vertex not belonging to any handle,

(4) the number of teeth that each handle intersect is odd and at least three, 


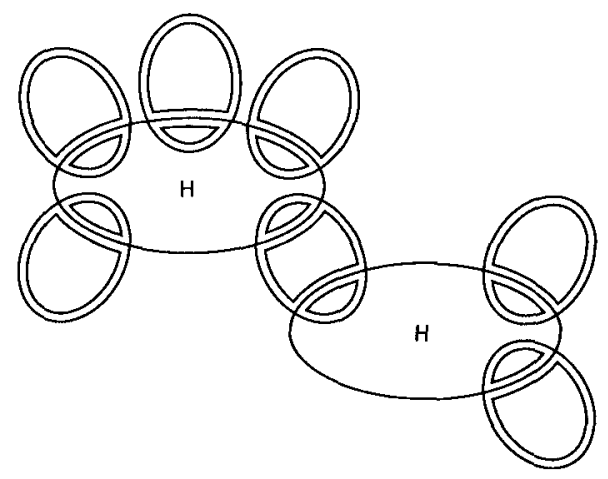

Fig. 1. A clique tree with two handles (indicated by an $\mathrm{H}$ ) and seven teeth. The closed curves correspond to the sets $S$ with $y_{S}=\frac{1}{2}$ in the proof of Theorem 4 .

(5) the intersection graph of the handles and teeth is a tree.

A clique tree is represented in Fig. 1. When the number of handles $h$ is 1 , the clique tree is called a comb. Combs were introduced by Chvátal [5] for the case with at most one common vertex between a tooth and a handle and by Grötschel and Padberg [15] for the general case. The clique tree inequality (in tight triangular form) is

$$
\sum_{i=1}^{h} x\left(\delta\left(H_{i}\right)\right)+\sum_{i=1}^{t} x\left(\delta\left(T_{i}\right)\right) \geqslant 2 \sum_{i=1}^{t} t_{i}+t+1
$$

where $t_{i}$ denotes the number of handles intersecting $T_{i}$. For simplicity, let $p=\sum_{i=1}^{t} t_{i}$. Since the intersection graph of the handles and teeth is a tree with $t+h$ vertices and $p$ edges, we have $p=t+h-1$. Hence, the clique tree inequality can be rewritten as

$$
\sum_{i=1}^{h} x\left(\delta\left(H_{i}\right)\right)+\sum_{i=1}^{t} x\left(\delta\left(T_{i}\right)\right) \geqslant 3 t+2 h-1 .
$$

Clique tree inequalities define facets of GTSP or STSP [17].

Theorem 4. The strength of a clique tree inequality with $h$ handles and $t$ teeth is at most

$$
\frac{3 t+2 h-1}{3 t+h-1} .
$$

From the above theorem, we can derive the following corollary.

\section{Corollary 5.}

(1) The strength of a comb inequality with $t$ teeth is at most $(3 t+1) / 3 t$;

(2) the strength of a comb inequality is at most $\frac{10}{9}$;

(3) the strength of a clique tree inequality with h handles is at most $(8 h+2) /(7 h+2)$;

(4) the strength of a clique tree inequality is at most $\frac{8}{7}$. 
Items (1) and (2) follow from the definition of combs, item (3) from the fact that $t \geqslant 2 h+1$ (since the number of edges incident to handles in the intersection graph is at least $3 h$ and at most $p=t+h-1$ ), and item (4) from item (3).

Proof of Theorem 4. From the discussion in Section 3.1, we need to construct a feasible solution for (D) of value $3 t+h-1$. Our dual solution is the following. Let $y_{S}=\frac{1}{2}$ for any $S$ of the form

$$
S= \begin{cases}H_{i} \cap T_{j}, & 1 \leqslant i \leqslant h, 1 \leqslant j \leqslant t \text { and } H_{i} \cap T_{j} \neq \emptyset, \\ T_{j}, & 1 \leqslant j \leqslant t, \\ T_{j}-\bigcup_{i} H_{i}, & 1 \leqslant j \leqslant t,\end{cases}
$$

and $y_{S}=0$ otherwise. (See Fig. 1 for an illustration of $y$.) Notice that, by definition of a clique tree, all these sets are nonempty. Also,

$$
2 \sum_{S} y_{S}=\sum_{i=1}^{t} t_{i}+2 t=p+2 t=3 t+h-1 .
$$

Finally, one can easily verify that $y$ is a dual feasible solution, completing the proof.

Remark. Using a result of [4], the strength of a clique tree inequality can be shown to be exactly equal to

$$
\frac{3 t+2 h-1}{3 t+2 h-2-\mathrm{npt}}
$$

where npt denotes the number of nonpendent teeth of the clique tree. A nonpendent tooth is a tooth intersecting more than one handle. Indeed, it is easy to derive from [4, Theorem 2.2] that $Z_{\mathrm{SP}}=3 t+2 h-2-n p t$, where $Z_{\mathrm{SP}}$ denotes the optimum value when optimizing over SP. Notice that this is in agreement with Theorem 4 since one can easily show (for example, by induction) that npt $\leqslant h-1$. This also shows that our upper bounds on the strength of subclasses of clique tree inequalities described in Theorem 4 and Corollary 5 can be attained.

\subsection{Path inequalities}

Path inequalities were introduced in [7]. A $k$-path configuration is defined by an odd integer $k \geqslant 3$, integers $n_{i} \geqslant 2$ for $i=1, \ldots, k$ and a partition of the vertex set $V$ into $\left\{A, Z, B_{j}^{i}\right.$ for $i=1, \ldots, k$ and $\left.j=1, \ldots, n_{i}\right\}$ where $A$ and/or $Z$ could be empty. For notational convenience, let $B_{0}^{i}=A$ and $B_{n_{i}+1}^{i}=Z$ for $i=1, \ldots, k$. Let $\delta(S, T)$ be $\delta(S) \cap \delta(T)$. The $k$-path inequality is $a x \geqslant a_{0}$ where

$$
a_{0}=1+\sum_{i=1}^{k} \frac{n_{i}+1}{n_{i}-1}
$$




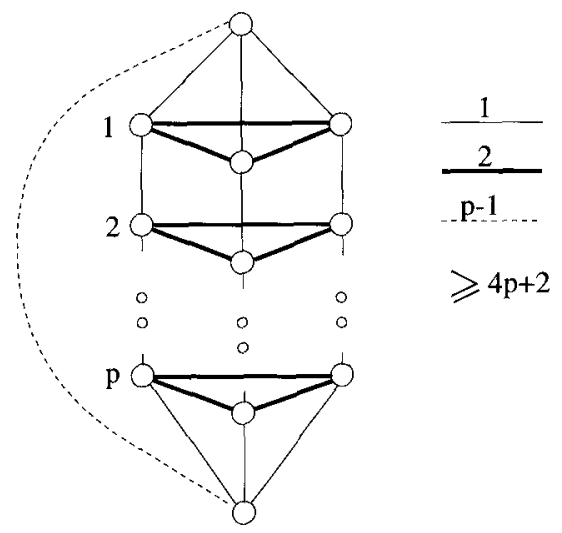

Fig. 2. A p-regular 3-path inequality. The coefficient $a_{e}$ in the inequality $a x \geqslant 4 p+2$ is equal to the shortest path length between the endpoints of $e$ in the above weighted graph.

$$
a_{e}=\left\{\begin{array}{lc}
1, & e \in \delta(A, Z), \\
\frac{|j-l|}{n_{i}-1}, & e \in \delta\left(B_{j}^{i}, B_{l}^{i}\right), i=1, \ldots, k \text { and } \\
\frac{1}{n_{h}-1}+\frac{1}{n_{i}-1}+\left|\frac{j-1}{n_{h}-1}-\frac{l-1}{n_{i}-1}\right|, & e \in \delta\left(B_{j}^{h}, B_{l}^{i}\right), j=1, \ldots, n_{h}, \\
0, & h \neq i \text { and } l=1, \ldots, n_{i}, \\
\text { otherwise. }
\end{array}\right.
$$

When $n_{i}=p$ for all $i$, the $k$-path inequality is called $p$-regular and takes a particularly attractive form as can be seen in Fig. 2 for the case $k=3$. The class of 2-regular path inequalities is equivalent to the class of comb inequalities. The $k$-path inequalities are facet-defining for GTSP [7].

Theorem 6. The strength of a path inequality is

$$
\frac{1+\sum_{i=1}^{k} r_{i}}{\sum_{i=1}^{k} r_{i}} \leqslant \frac{k+1}{k} \leqslant \frac{4}{3},
$$

where $r_{i}=\left(n_{i}+1\right) /\left(n_{i}-1\right)$.

Proof. Let $y$ be defined by (see Fig. 3):

$$
y_{S}= \begin{cases}\frac{1}{2} \frac{1}{n_{i}-1}, & S=B_{j}^{i} \text { with } 1 \leqslant i \leqslant k \text { and } 1 \leqslant j \leqslant n_{i}, \\ \frac{1}{2} \frac{1}{n_{i}-1}, & S=\bigcup_{j=1}^{n_{i}} B_{j}^{i} \text { with } 1 \leqslant i \leqslant k, \\ 0, & \text { otherwise. }\end{cases}
$$

One can verify that $y$ is a feasible solution for (D) with value $2 \sum_{S} y_{S}=\sum_{i=1}^{k} r_{i}$. Moreover, $y$ is an optimal solution since a primal solution of the same value can be 


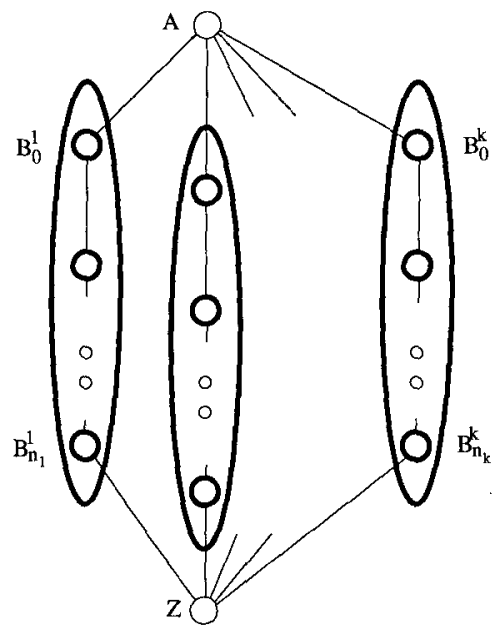

Fig. 3. Illustration of the dual solution $y$ in the case of a path configuration inequality. $y$ has a nonzero component only for those sets represented as thick circles or ellipses.

obtained by selecting an edge from each set $\delta\left(B_{j}^{i}, B_{j+1}^{i}\right)$ for $i=1, \ldots, k$ and $j=$ $0, \ldots, n_{i}$ as well as edges from within the $B_{j}^{i}$. Using the arguments of Section 3.1 , the strength of a path inequality is thus equal to

$$
\frac{1+\sum_{i=1}^{k} r_{i}}{\sum_{i=1}^{k} r_{i}}=1+\frac{1}{\sum_{i=1}^{k} r_{i}} \leqslant 1+\frac{1}{k} \leqslant \frac{4}{3},
$$

where we have used the facts that $r_{i} \geqslant 1$ and $k \geqslant 3$.

\subsection{Crown inequalities}

Crown inequalities were recently discovered in [24]. Consider a partition of $V$ into $4 k$ $(k \geqslant 2)$ vertex sets $U_{1}, \ldots, U_{4 k}$. Given $1 \leqslant i, j \leqslant 4 k$, let $\langle i, j\rangle$ be defined as the distance on the cycle $\{1,2, \ldots, 4 k, 1\}$, i.e., $\langle i, j\rangle=|i-j|$ if $|i-j| \leqslant 2 k$ and $\langle i, j\rangle=4 k-|i-j|$,

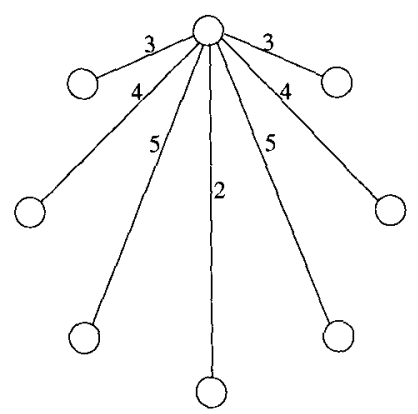

Fig. 4. Crown inequality on eight vertices $(k=2)$. The right-hand side is 22 and the coefficients of all edges can be obtained by simply rotating the edges of the figure. 
otherwise. The crown inequality is the inequality $a x \geqslant a_{0}$ where $a_{0}=12 k(k-1)-2$ and

$$
a_{e}= \begin{cases}4 k-6+\langle i, j\rangle, & e \in \delta\left(U_{i}, U_{j}\right), \quad 1 \leqslant\langle i, j\rangle \leqslant 2 k-1, \\ 2 k-2, & e \in \delta\left(U_{i}, U_{i+2 k}\right), \quad 1 \leqslant i \leqslant 2 k, \\ 0, & \text { otherwise. }\end{cases}
$$

The simplest crown inequality is represented in Fig. 4. The crown inequalities are facet-defining for $k \geqslant 2[24]$.

Theorem 7. The crown inequality has strength

$$
\frac{6 k(k-1)-1}{6 k(k-1)-k} \leqslant \frac{11}{10} \text {. }
$$

Proof. As usual, we claim that the following vector $y$ is a feasible solution to (D) of value $12 k(k-1)-2 k$ :

$$
y_{S}= \begin{cases}k-1, & S=U_{i} \text { with } 1 \leqslant i \leqslant 4 k, \\ k-\frac{3}{2}, & S=U_{i} \cup U_{i+2 k} \text { with } 1 \leqslant i \leqslant 2 k, \\ 0, & \text { otherwise. }\end{cases}
$$

This vector $y$ is optimal since a primal solution $x$ of the same value can be derived: set $x_{e}=1$ for one edge $e$ from each diameter $\delta\left(U_{i}, U_{i+2 k}\right), x_{e}=0.5$ for one edge $e$ from each $\delta\left(U_{i}, U_{i+1}\right)$ and also $x_{e}=1$ for edges within $U_{i}$.

\subsection{Hypohamiltonian inequalities}

A hypohamiltonian graph is a nonhamiltonian graph such that the deletion of any node yields a hamiltonian graph. The Petersen graph is the smallest hypohamiltonian graph (Sosselier, see [2]). Consider a partition of $V$ into $V_{1}, \ldots, V_{k}$ and let $H$ be an edge maximal hypohamiltonian graph with vertex set $\{1, \ldots, k\}$. The resulting hypohamiltonian inequality is $a x \geqslant a_{0}$ where $a_{0}=k+1$ and

$$
a_{e}= \begin{cases}1, & e \in \delta\left(V_{i}, V_{j}\right), \quad i \neq j \text { and }(i, j) \in H, \\ 2, & e \in \delta\left(V_{i}, V_{j}\right), \quad i \neq j \text { and }(i, j) \notin H, \\ 0, & \text { otherwise. }\end{cases}
$$

The hypohamiltonian inequalities were shown to be facet-defining for GTSP in [7]. For related results for STSP, see [16].

Theorem 8. The strength of a hypohamiltonian inequality corresponding to a hypohamiltonian graph on $k$ vertices is at most

$$
\frac{k+1}{k} \leqslant \frac{11}{10} .
$$

Proof. Let $y_{S}=\frac{1}{2}$ for any $S$ of the form $V_{i}$ and $y_{S}=0$ otherwise. Clearly, $y$ is a feasible solution to (D) of value $k$. Hence, an upper bound on the strength is $(k+1) / k$. This 
is at most $\frac{11}{10}$ since the smallest hypohamiltonian graph is the Petersen graph (on ten vertices).

\subsection{Miscellany}

As we have seen, it is often child's play to compute the strength (or at least an upper bound on the strength) of an inequality for GTSP. The reader can experiment with his own favorite class of inequalities. As a last example, one can easily show that a planted clique tree inequality as defined in [23] has strength upper bounded by

$$
\frac{4 t+2 h}{4 t+h-1} \leqslant \frac{10 h+4}{9 h+3}
$$

This is at most $\frac{8}{7}$ for $h \geqslant 2$. For $h=1$, the planted clique tree inequality reduces to the 3-regular 3-path inequality whose strength is equal to $\frac{7}{6}$ in agreement with Theorem 6 .

\section{Interpretation}

We give three different interpretations of the results of the previous section.

(1) Experimental results show that strong cutting-plane algorithms work very well for the travelling salesman problem. This seems to indicate that the intersection of all the known classes of inequalities for STSP or GTSP approximates it very closely. The results of the previous sections show that if one optimizes any nonnegative cost function over all subtour elimination constraints, all clique tree inequalities, all path inequalities, all crown inequalities, all hypohamiltonian inequalities and all planted clique tree inequalities, the resulting bound is at most $\frac{4}{3}$ times the Held-Karp lower bound. This is some strongly supporting evidence for Conjecture 1 .

(2) If the clique tree inequalities and the subtour elimination constraints are sufficient to prove optimality for some instance of the TSP, then the Held--Karp lower bound must be within a factor of $\frac{8}{7}<115 \%$ of optimal. This gives some explanation of the tightness of the bound in practice.

(3) If one considers a special instance of the travelling salesman problem in which the cost function consists of the left-hand side of a $p$-regular 3-path inequality with $p$ large, then all the classes of valid inequalities studied in this paper, except the path inequalities themselves, will do badly on this instance. Indeed, the class of comb inequalities for example will close at most

$$
\frac{\frac{10}{9}-1}{\frac{4}{3}-1}=\frac{1}{3}=33.3 \%
$$

of the duality gap. This is not impressive. Similarly the class of clique trees will close at most

$$
\frac{\frac{8}{7}-1}{\frac{4}{3}-1}=\frac{3}{7}<43 \%
$$


of the duality gap. In fact, to close more than $43 \%$ of the duality gap, one must use the path inequalities, or some class of inequalities which is not considered in this paper.

\section{Concluding remarks}

The results of the previous sections apply to GTSP. One might wonder whether the worst-case improvements remain unchanged when one adds the degree constraints $x(\delta\{i\})=2$ for all $i \in V$ and restricts one's attention to cost functions satisfying the triangle inequality. We believe so but have been unable to prove it. The result would follow immediately if one could prove that the degree constraints never affect the value of the relaxation when the cost function satisfies the triangle inequality. In the two extreme cases (the subtour polytope and STSP), this is indeed correct (for the subtour polytope, see [11]).

In this paper, we have provided a measure to evaluate relaxations for GTSP. It would be worthwhile to investigate other measures. In particular, one could try to compare the volumes of various relaxations for the TSP.

Finally, since the TSP is NP-hard, one should not expect to obtain a simple description of the facets of GTSP. Nevertheless, one could hope for the derivation of properties of them which allow to prove an upper bound on the strength of any facet-defining valid inequality. This could lead to a proof of Conjecture 1 .

\section{References}

[1] D. Applegate, R. Bixby, V. Chvátal and W. Cook, "Finding cuts in the TSP," DIMACS Technical Report 95-0S, Rutgers University, New Brunswick, NJ, 1995.

[2] C. Berge, Graphes et Hypergraphes (Dunod, Paris, 1970).

[3] S. Boyd and W. Cunningham, "Small traveling salesman polytopes," Mathematics of Operations Research 16 ( 1991 ) 259-271.

[4] S.C. Boyd and W.R. Pulleyblank, "Optimizing over the subtour polytope of the travelling salesman problem," Mathematical Programming 49 (1991) 163-187.

[5] V. Chvátal, "Edmonds polytopes and weakly Hamiltonian graphs," Mathematical Programming 5 (1973) $29-40$.

[6] J.-M. Clochard and D. Naddef, "Using path inequalities in a branch and cut code for the traveling salesman problem," in: G. Rinaldi and L.A. Wolsey, eds., Proceedings of the 3rd Integer Programming and Combinatorial Optimization Conference (CORE, Louvain-la-Neuve, 1993) pp. 291-311.

[7] G. Cornuéjols, J. Fonlupt and D. Naddef, "The traveling salesman problem on a graph and some related integer polyhedra," Mathematical Programming 33 (1985) 1-27.

[8] H. Crowder and M. Padberg, "Solving large-scale symmetric traveling salesman problems to optimality," Management Science 26 (1980) 495-509.

[9] B. Fleischmann, "A new class of cutting planes for the symmetric traveling salesman problem," Mathematical Programming 40 (1988) 225-246.

[10] J. Fonlupt and D. Naddef, "The traveling salesman problem in graphs with some excluded minors," Mathenatical Programming 53 (1992) 147-172.

[11] M.X. Goemans and D. Bertsimas, "Survivable networks, linear programming relaxations and the parsimonious property," Mathematical Programming 60 (1993) 145-166.

[12] M. Grötschel, "On the monotone symmetric travelling salesman problem: hypohamiltonian/hypotraceable graphs and facets," Mathematics of Operations Research 5 (1980) 285-292. 
[13] M. Grötschel, "On the symmetric traveling salesman problem: solution of a 120-city problem," Mathematical Programming Study 12 (1980) 61-77.

[14] M. Grötschel and O. Holland, "Solution of large-scale symmetric traveling salesman problems," Mathematical Programming 51 (1991) 141-202.

[15] M. Grötschel and M.W. Padberg, "On the symmetric travelling salesman problem I: inequalities," Mathematical Programming 16 (1979) 265-280.

[16] M. Grötschel and M.W. Padberg, "Polyhedral theory," in: E. Lawler, J. Lenstra, A. Rinnooy Kan and D. Shmoys, eds., The Traveling Salesman Problem: A Guided Tour of Combinatorial Optimization (Wiley, New York, 1985) pp. 251-305.

[17] M. Grötschel and W.R. Pulleyblank, "Clique tree inequalities and the symmetric traveling salesman problem," Mathematics of Operations Research 11 (1986) 537-569.

[18] M. Held and R.M. Karp, "The traveling-salesman and minimum cost spanning trees" Operations Research 18 (1970) 1138-1162.

[19] M. Jünger, G. Reinelt and S. Thienel, "Provably good solutions for the traveling salesman problem," Report 92.114, Institut für Informatik, Universität zu Köln, 1992.

[20] J. Lee and W.D. Morris, "Geometric comparison of combinatorial polytopes," CORE Discussion Paper 9216, CORE, Université Catholique de Louvain, 1992.

[21] C. Monma, B. Munson and W. Pulleyblank, "Minimum-weight two-connected spanning networks," Mathematical Programming 46 (1990) 153-171.

[22] D. Naddef, "The binested inequalities for the symmetric traveling salesman polytope," Mathematics of Operations Research 17 (1992) 882-900.

[23] D. Naddef and G. Rinaldi, "The symmetric traveling salesman polytope and its graphical relaxation: Composition of valid inequalities," Mathematical Programming 51 (1991) 359-400.

[24] D. Naddef and G. Rinaldi, "The crown inequalities for the symmetric traveling salesman polytope," Mathematics of Operations Research 17 (1992) 308-326.

[25] D. Naddef and G. Rinaldi, "The graphical relaxation: A new framework for the symmetric traveling salesman polytope," Mathenatical Programming 58 (1993) 53-87.

[26] M.W. Padberg and S. Hong, "On the symmetric travelling salesman problem: A computational study," Mathenatical Programming Study 12 (1980) 78-107.

[27] M. Padberg and G. Rinaldi, "Facet identification for the symmetric traveling salesman polytope," Mathenatical Programming 47 (1990) 219-257.

[28] M. Padberg and G. Rinaldi, "A branch-and-cut algorithm for the resolution of large-scale symmetric traveling salesman problems," SIAM Review 33 (1991) 60-100.

[29] W. Pulleyblank, "Polyhedral combinatorics," in: G. Nemhauser, A.H.G. Rinnooy Kan and M.J. Todd, eds., Optimization, Handbooks in Operations Research and Management Science, Vol. 1 (North-Holland, Amsterdam, 1989) pp. 371-446.

[30] D.B. Shmoys and D.P. Williamson, "Analyzing the Held-Karp TSP bound: A monotonicity property with application," Information Processing Letters 35 (1990) 281-285.

[31] L.A. Wolsey, "Heuristic analysis, linear programming and branch and bound," Mathematical Programming Study 13 (1980) 121-134. 